\title{
Predicción del deterioro de los componentes metálicos de una Estación Reductora de Presión implementando el método de la cadena de Márkov
}

\author{
FERNEy CAMilo JimÉNEZ BeLtrán ${ }^{1}$ \\ Milton Mena SERna ${ }^{2}$ \\ CÉSAR Augusto García-UbaQuE ${ }^{2}$
}

\section{Resumen}

Una Estación Reductora de Presión (ERP) sirve para controlar la presión de servicio y garantizar el correcto funcionamiento de las redes del acueducto en un sector determinado. En la estructura de las ERPs, las tuberías y los accesorios de acero están expuestos a la corrosión y por ende a un deterioro más rápido. Es por ello que, basado en las condiciones actuales se planteó un método para predecir este deterioro, y de esta manera, lograr un mejor manejo al mantenimiento y rehabilitación de estos elementos.

Palabras claves: Probabilidad, Deterioro, Corrosión, Distribución de agua, Mantenimiento.

\section{Deterioration prediction of metallic components of a Pressure Regulatory Station by implementing the Markov chain method}

\section{Abstract}

A Pressure Reducing Station (ERP) serves to control the service pressure and ensure the correct operation of aqueduct networks in a given sector. In the ERPs, structure, the steel pipes and fittings are exposed to corrosion and therefore to faster deterioration. That is why, based on current conditions, a method was proposed to predict this deterioration, and achieve a better maintenance management and rehabilitation of these elements.

Keywords: Probability, Deterioration, Corrosion, Water distribution, Maintenance..

1 Empresa de Acueducto y Alcantarillado de Bogotá, Colombia.

2 Universidad Distrital Francisco José de Caldas, Bogotá, Colombia.

Autor de correspondencia: García-Ubaque, C.A. (César Augusto): Universidad Distrital Francisco José de Caldas: Calle 68 D Bis A Sur \# 49 F - 70 Bloque 6, piso 1, Bogotá-Colombia. Teléfono: (1) 3239300. Correo electrónico: cagarciau@ udistrital.edu.co
Historia del artículo:

Artículo recibido: 16-VII-2019/ Aprobado: 15-I-2020

Disponible online: 06 de abril de 2020

Discusión abierta hasta septiembre de 2021 


\section{Previsão da deterioração dos componentes metálicos de uma Estação Redutora de Pressão através da implementação do método da cadeia Márkov}

\section{Resumo}

Uma Estação Redutora de Pressão (ERP) serve para controlar a pressão de serviço e garantir a operação correta das redes de aquedutos em um determinado setor. Na estrutura dos ERPs, os tubos e conexões de aço estão expostos à corrosão e, portanto, a uma deterioração mais rápida. É por isso que, com base nas condições atuais, foi proposto um método para prever essa deterioração e, assim, obter um melhor gerenciamento da manutenção e reabilitação desses elementos.

Palavras chaves: Probabilidade, Deterioração, Corrosão, Distribuição de água, Manutenção..

\section{Introducción}

El deterioro progresivo de un metal provocado por la reacción entre el material y el medio que lo rodea se define como corrosión húmeda (Empresa de Acueducto y Alcantarillado de Bogotá, 2006). Esta se presenta generalmente en medios como el agua y afectan en gran medida el funcionamiento de estructuras que se encuentran expuestas continuamente, como es el caso de las Estaciones reductoras de presión (ERP) y estructuras de control de presión, utilizadas en los sistemas de distribución de agua potable con la finalidad de mantener una presión definida en cierta zona de consumo (Bai, et al., 2018). Estas están compuestas por accesorios, niples y válvulas en acero y hierro dúctil (Empresa de Acueducto y Alcantarillado de Bogotá, 2003), materiales que al estar expuestos a la humedad se deterioran y se corroen lenta y progresivamente (Mamlouk, 2009) (Ren, et al., 2018).

En la práctica, muchas veces no se tiene en cuenta este fenómeno, lo cual hace que las empresas prestadoras de este servicio permitan el avance de corrosión dichas estructuras, y solo intervienen cuando presentan fallas o dejan de funcionar. Además, omiten mantenimientos periódicos que ayudarían a prolongar el funcionamiento y a optimizar los recursos asignados a esta área (IRIM, 2018) (Opel, et al., 2018). Asimismo, las empresas no han logrado implementar en su totalidad metodologías que permitan garantizar con un mayor grado de certeza el deterioro de los elementos de las ERPs.

Por lo anterior, este estudio tiene como objetivo estimar el estado futuro del deterioro de los componentes metálicos de una ERP mediante la aplicación del método de la cadena de Markov, utilizando datos reales provenientes de inspecciones rutinarias a las ERPs en el Acueducto de Bogotá.

\section{Metodología}

\section{Método de la cadena de Markov}

Existen diferentes métodos para predecir el deterioro en estructuras. Uno de estos métodos es la función del ratio de fallo que representa la incertidumbre sobre el tiempo de fallo de una estructura, esta incertidumbre se incrementa con el paso del tiempo pasando por diferentes estados de degradación. Este método resulta poco interesante puesto que solo ofrece el valor de la probabilidad de fallo teniendo en cuenta unos objetivos preestablecidos.

Existen otros métodos de deterioro basados en procesos estocásticos, los cuales dependen directamente del tiempo, algunos de los procesos estocásticos más utilizados para la modelación del deterioro son el proceso Gamma y el proceso Gaussiano. El primero se caracteriza por presentar incrementos independientes no negativos representados por una función de distribución Gamma. Por su parte el segundo se basa en 
el movimiento Browniano, en este la resistencia de la estructura con el paso del tiempo aumenta y disminuye alternativamente. Así mismo, podemos encontrar el modelo de Rijkswaterstaat en el cual el envejecimiento de la estructura está modelado mediante un proceso gamma.

Finalmente podemos encontrar el método en el que basó la investigación, el método de la cadena de Márkov se basa en un proceso estocástico, donde la condición futura solo depende de la condición actual del elemento (Tang, et al., 2015) (Bedoya \& Barrera, 2006). Para aplicar este método se considera que el proceso de corrosión es continuo (Micevski, et al., 2002), por consiguiente, el estado de corrosión no puede regresar a un estado anterior siempre y cuando no se alteren las condiciones en las que funcionan comúnmente los elementos, tal como se muestra en la Figura 1.

\section{Figura 1. Representación gráfica del método de Márkov.}

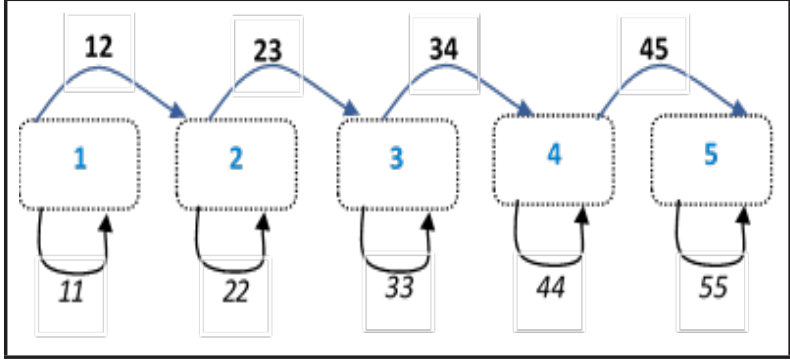

A diferencia de los otros métodos, la gran ventaja del método de Markov es que expresa sus predicciones de la misma manera en la que se haría normalmente en las inspecciones de una estructura, al estar basado en unos estados de conservación, es fácil de adaptar a los datos obtenidos de una inspección visual y permite incorporar nuevas inspecciones en el tiempo (Clemente, 2003) (Arafin \& Szpunar, 2009).

\section{Recolección de datos}

Para este estudio se utilizaron registros y reportes de mantenimientos efectuados en las ERPs de las zonas de servicio 1 y 4 de la Empresa de Acueducto y Alcantarillado de Bogotá. Estas zonas comprenden las localidades del norte: Suba y Usaquén y sur de la ciudad: San Cristóbal, Usme, Tunjuelito, Kennedy, Puente Aranda, Rafael Uribe Uribe y Ciudad Bolívar, respectivamente. Se revisaron y analizaron 41 registros fotográficos, a continuación, se muestra una de las estaciones que se analizaron.
Figura 2. Estación reguladora de presión ubicada en la Zona 1, EAB.

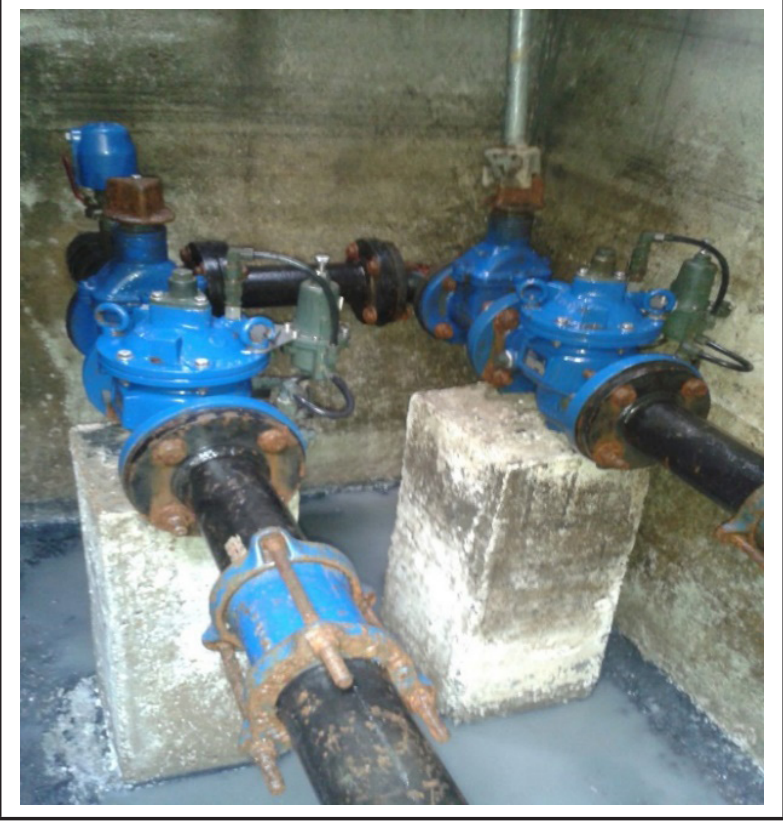

Definición de las etapas de deterioro

Para clasificar la condición de deterioro en las estructuras metálicas se utilizó la escala propuesta por (Saucer \& Riveros, 2009), la cual comprende 5 etapas de deterioro, la primera representa un estado óptimo, y la última, la condición de daño total (Sinha, et al., 2010). A continuación, se describirán brevemente cada uno de estos estados de los componentes metálicos:

1. Estado óptimo: Están protegidos por un revestimiento total, se encuentran en un estado nuevo.

2. Corrosión imperceptible: Presentan pequeñas zonas con deterioro por corrosión o desprendimiento del recubrimiento, no se puede medir fácilmente.

3. Corrosión leve: en una parte de los componentes se presenta deterioro moderado posible de medir, no obstante, esta condición no impide su correcto funcionamiento.

4. Corrosión grave: en gran parte de los elementos se presenta varias formas de corrosión, pérdidas de espesor y carencia de recubrimiento, generando disminución de la capacidad estructural e hidráulica.

5. Daño total: los elementos son afectados totalmente, requieren cambio inmediato. 


\section{Clasificación de los datos}

Los estados de corrosión previamente definidos se asignaron mediante la observación detallada por parte de expertos de la Empresa del Acueducto de Bogotá. A continuación, se muestran los datos obtenidos.

\section{TABLA 1. RECOPILACIÓN Y CLASIFICACIÓN DE DATOS}

\begin{tabular}{|c|c|c|c|c|c|c|c|c|c|}
\hline & & 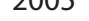 & 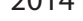 & 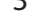 & \\
\hline \multirow{3}{*}{ ERP } & \multirow{3}{*}{$\begin{array}{c}\text { Año de } \\
\text { instalación }\end{array}$} & \multirow{3}{*}{$\begin{array}{c}\text { Año de } \\
\text { inspección }\end{array}$} & \multirow{3}{*}{$\begin{array}{l}\text { Tipo de } \\
\text { daño }\end{array}$} & \multirow{3}{*}{$\begin{array}{l}\text { Años de } \\
\text { servicio }\end{array}$} & 38 & 2005 & 2014 & 3 & 9 \\
\hline & & & & & 39 & 2005 & 2014 & 3 & 9 \\
\hline & & & & & 40 & 2005 & 2017 & 3 & 12 \\
\hline 1 & 2008 & 2014 & 3 & 6 & 41 & 2005 & 2017 & 4 & 12 \\
\hline
\end{tabular}

\section{Aplicación del modelo de Markov}

\section{Derivación de las probabilidades de transición:}

Inicialmente se calculó la media aritmética a todos los registros recopilados con el fin de determinar la línea de tendencia logarítmica, esto permitió encontrar los estados de corrosión en el tiempo con datos actuales, como aparece en la Figura 3.

Figura 3. Estados de corrosión de los componentes metálicos

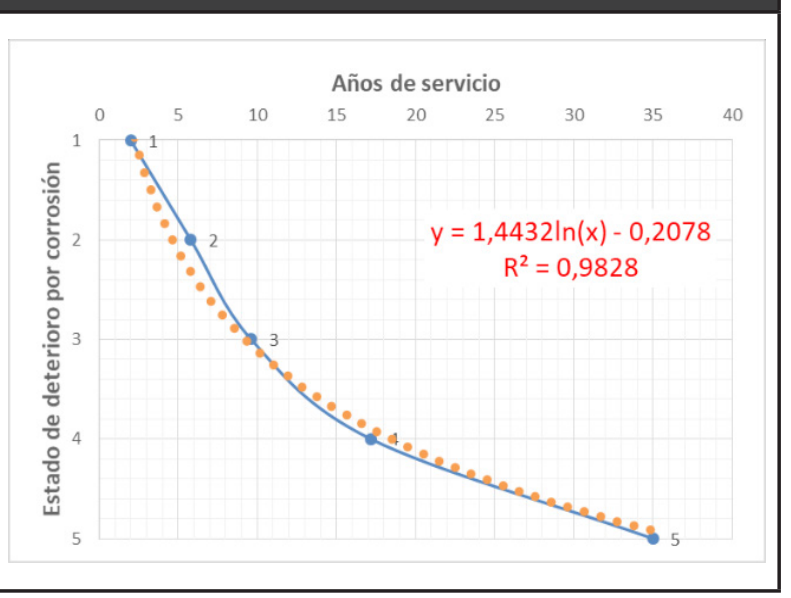

Usando la distribución Weibull se determinaron valores aleatorios para simular los estados de condición cada 5 años hasta llegar a los 35 años (vida útil de las ERPs). Luego se determinó los valores de probabilidad de transición (Lara, s.f.) de los estados de condición como se muestra en la Tabla 2. 


TABLA 2. MATRIZ DE TRANSICIÓN
\begin{tabular}{c|c|c|c|c|c}
\hline $\begin{array}{c}\text { Estados de } \\
\text { condición }\end{array}$ & $\mathbf{1}$ & $\mathbf{2}$ & $\mathbf{3}$ & $\mathbf{4}$ & $\mathbf{5}$ \\
\hline 1 & $1,8 \%$ & $98,2 \%$ & 0 & 0 & 0 \\
\hline 2 & 0 & $48,1 \%$ & $51,9 \%$ & 0 & 0 \\
\hline 3 & 0 & 0 & $9,9 \%$ & $90,1 \%$ & 0 \\
\hline 4 & 0 & 0 & 0 & $43,9 \%$ & $56,1 \%$ \\
\hline 5 & 0 & 0 & 0 & 0 & $100,0 \%$ \\
\hline
\end{tabular}

Vectores de estados iniciales y de condición:

Para este método se determinaron los estados iniciales y los estados de condición vectorialmente mediante la Ecuación 1 y la Ecuación 2 de la siguiente manera:

$$
\begin{aligned}
& Q_{0}=[1,0,0,0,0] \\
& R=\left[\begin{array}{lllll}
1 & 2 & 3 & 4 & 5
\end{array}\right]
\end{aligned}
$$

A partir de la matriz de transición y los vectores mencionados anteriormente se estimaron las probabilidades de los estados de corrosión en el futuro mediante las operaciones matriciales que aparecen a continuación (Ecuación 3, Ecuación 4 y Ecuación 5):

$$
\begin{gathered}
P_{t}=P^{t} \\
Q_{t}=Q_{0} * P^{t} \\
R_{p t}=Q_{t} * R^{T}
\end{gathered}
$$

Siendo la Ecuación 3 la probabilidad del estado a futuro en el tiempo t; la Ecuación 4 el vector $Q_{t}$ que representa el estado después de t periodos y la Ecuación 5 el estado de condición $R_{p t^{\prime}}$ después de t periodos.

\section{Resultados y discusión}

Después de aplicar el método, se generó la figura 4 donde se presenta la curva del deterioro por corrosión en una Estación Reguladora de Presión en el Acueducto de Bogotá. En esta gráfica se muestran los resultados hasta los 35 años con intervalos de 5 años.

Adicionalmente se muestra la línea de la media aritmética de los datos recopilados y la línea de interpolación logarítmica. Estas se muestran con el fin de compararlas y verificar que el método nos arroja resultados con comportamientos acertados.

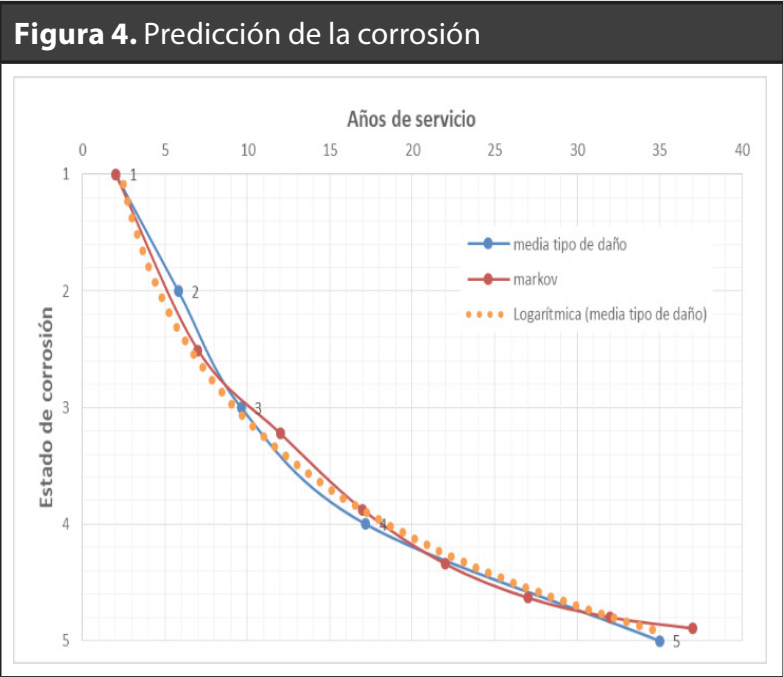

De la gráfica anterior se encontró que el estado de corrosión 2 ocurre aproximadamente a los 5 años de uso, el estado 3 a los 10 años de servicio y los estados 4 y 5 se presentan a los 18 y 38 años respectivamente. Dado lo anterior, se evidencian cambios de estados significativamente más rápidos en los primeros años con relación a los últimos años, donde este proceso se fue reduciendo.

\section{Validación del método}

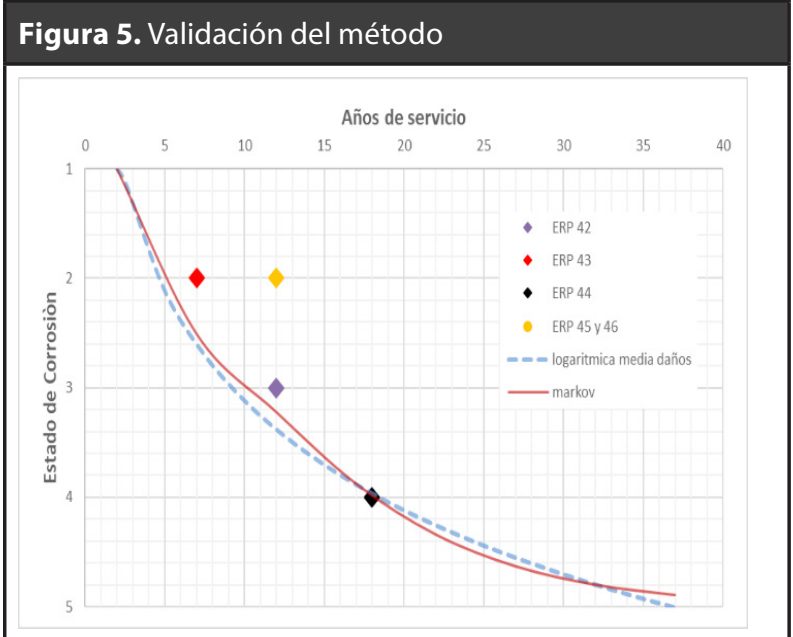

Con el fin de verificar los resultados obtenidos en el método aplicado, se recolectó la información de cinco ERPs adicionales, ubicadas en otras zonas de servicio de la Empresa de Acueducto y Alcantarillado de Bogotá. Con lo anterior, se realizó la comparación entre estos datos y los resultados del método de 
Márkov, evidenciándose que el método permite predecir adecuadamente el estado de deterioro de las ERPs en la mayoría de los casos. Sin embargo, se observó que algunas estaciones presentaron desviaciones debido al grado de incertidumbre de clasificación de deterioro. Dicha comparación se muestra en la Figura 5.

\section{Conclusiones}

El método utilizado para la predicción del deterioro de los componentes metálicos de una Estación Reguladora de Presión y sus resultados demuestran confiabilidad ya que presentan similitud con las curvas y datos de referencia.

La información obtenida permitirá a las diferentes empresas de acueducto tener una línea de referencia para poder comparar con los datos obtenidos en campo, y tomar las medidas necesarias en el momento justo. De la misma manera, podremos determinar si el deterioro está avanzando adecuadamente, o en el peor de los casos, de una manera más rápida.

De los resultados obtenidos se sugieren dos tipos de mantenimiento: preventivo y correctivo. El mantenimiento preventivo cuando el deterioro por corrosión no supere el estado de corrosión 3 o cuando se aproxime a los 20 años de servicio. También, se aconseja realizar mantenimientos correctivos e inspecciones más especializadas cuando se presenten estados de corrosión 4. Por último, se recomienda el cambio inmediato cuando los elementos muestren señales de estado de deterioro 5 o cuando supere los 30 años de servicio.

Para estudios futuros se podría implementar otras herramientas para la simulación de datos, tales como el método de Montecarlo, el algoritmo MetropolisHastings, o similares para la obtención de las probabilidades de transición de la cadena de Markov.

\section{Agradecimientos}

Agradecemos a la Empresa de Acueducto y Alcantarillado de Bogotá por habernos facilitado los datos, reportes y registros fotográficos que sirvieron como base para desarrollar esta investigación.

\section{Referencias}

Arafin, M. \& Szpunar, J., 2009. A Markov chain - Monte Carlo model for intergranular stress corrosion crack propagation in polyscrystalline materials. Materials, Science and Engineering, Volumen 513-514, pp. 254-266.

Bai, L., Jiang, K. \& Gao, L., 2018. The Influence and Mechanism of Residual Stress on the Corrosion Behavior of Welded Structures. Materials Research, jul.21(5).

Bedoya, J. \& Barrera, M., 2006. Convergencia de las cadenas de Markov. Scientia et Technica, 3(32).

Cabrera-de la Cruz, D., Patiño, C. \& Galván-Martínez, R., 2018. Análisis de la corrosión de uniones por soldadura fuerte de compósitos matriz cobre. Matéria (Río de Janeiro), jul.23(2).

Clemente, J. J., 2003. Modelos de predicción del deterioro en sistemas de gestión de puentes. Valencia: Universidad Politécnica de Valencia.

Empresa de Acueducto y Alcantarillado de Bogotá, 2003. Diseño de estaciones reductoras de presión para las redes de distribución de acueducto, Bogotá: EAAB.

Empresa de Acueducto y Alcantarillado de Bogotá, 2006. Protección de tuberías en redes de acueducto y alcantarillado, Bogotá: EAAB.

IRIM, 2018. Especial mantenimiento basado en condición. Madrid: RENOVETEC.

Lara, A. M., s.f. Universidad de Granada. [En línea] Available at: http://www.ugr. es/ bioestad/private/cpfund10.pdf [Último acceso: 3 septiembre 2018].

Mamlouk, M. S., 2009. Acero. En: Materiales para ingeniería civil. Madrid: Pearson Prentice Hall.

Micevski, T., Kuczera, G. \& Coombes, P., 2002. Markov model for storm water pipe. Journal of infraestructure systems, 8(2), pp. 49-56.

Opel, O. y otros, 2018. Corrosion in heating and cooling water circuits - A field study. Energy Procedia, Volumen 155, pp. 359-366.

Ren, L. y otros, 2018. Pipeline corrosion and leakage monitoring based on the distributed optical fiber sensing technology. Measurement, Volumen 122, pp. 57-95.

Saucer, P. W. \& Riveros, G. A., 2009. A system for collecting and compiling condition data for hidraulic steel structures for us in the assessment of risk and reliability an prioritization of manteinance and repairs. Washington D.C.: US Army Corp of Engineers.

Sinha, S. K., Angkasuwansiri, T. \& Thomasson, R., 2010. Background. En: Predicting the remaining economic lif of wastewater pipes. London: IWA Publishing. 
Tang, J., Brouste, A. \& Tsui, K., 2015. Some improvements of wind speed Markov chain modeling. Renewable Energy, Volumen 81, pp. 52-56.

PARA CITAR ESTE ARTÍCULO TO REFERENCE THIS ARTICLE / PARA CITAR ESTE ARTIGO /

Jiménez Beltrán, F.C. Mena Serna, M. García-Ubaque, C.A. (2020). Predicción del deterioro de los componentes metálicos de una Estación Reductora de Presión implementando el método de la cadena de Márkov. Revista EIA, 17(33) enero-junio, Reia33017 pág. 1-7. Disponible en: https://doi.org/10.24050/reia.v17i33.1342 